BA-08-22

\title{
NMSSM and Seesaw Physics at LHC
}

\author{
Ilia Gogoladze ${ }^{a}$, Nobuchika Okada ${ }^{b, c} 2_{2}$ and Qaisar Shafi, \\ ${ }^{a}$ Bartol Research Institute, Department of Physics and Astronomy, \\ University of Delaware, Newark, DE 19716, USA \\ ${ }^{b}$ Department of Physics, University of Maryland, College Park, MD 20742, USA \\ c Theory Group, KEK, Tsukuba 305-0801, Japan
}

\begin{abstract}
We consider extensions of the next-to-minimal supersymmetric model (NMSSM) in which the observed neutrino masses are described in terms of effective dimension six (or seven) rather than dimension five operators. All such operators respect the discrete symmetries of the model. The new particles associated with the double (or triple) seesaw mechanism can have sizable couplings to the known leptons, even with a $\mathrm{TeV}$ seesaw scale. In the latter case some of these new short-lived particles could be produced and detected at the LHC.
\end{abstract}

\footnotetext{
${ }^{1}$ E-mail: ilia@physics.udel.edu

On leave of absence from: Andronikashvili Institute of Physics, GAS, 380077 Tbilisi, Georgia.

${ }^{2}$ E-mail: okadan@post.kek.jp
} 
The next-to-minimal supersymmetric standard model (NMSSM) [1] provides a well motivated extension of MSSM in which the $\mu$ problem of the latter is resolved through the introduction of a gauge singlet superfield $S$. This (NMSSM) extension has several phenomenological consequences. For instance, the upper bound on the lightest (CP-even) MSSM Higgs scalar can be increased from around $125 \mathrm{GeV}$ to close to $140 \mathrm{GeV}$ [2], and the little hierarchy problem encountered in the MSSM is ameliorated [3, 4]. New Higgs boson decay channels into CP-odd scalars appear [4, 5, 6] which have an impact on Higgs boson searches, and there are new implications for dark matter physics [7, 8].

In order to incorporate the observed solar and atmospheric neutrino oscillations [9], we propose in this paper some extensions of the NMSSM based on the seesaw mechanism for providing neutrino masses. With the Large Hadron Collider (LHC) era about to unfold, we are especially interested in those seesaw extensions of the NMSSM which can be tested at the LHC. This leads us to consider dimension six and seven (rather than dimension five) operators for the generation of the observed neutrino masses and mixings. The presence of the $S$ VEV in the NMSSM turns out to be an important ingredient in implementing the double [10] (or triple) seesaw mechanism. We consider several possibilities for renormalizable models at high energies, which may include $\mathrm{SU}(2)$ singlet, triplet and even additional doublet chiral superfields.

For the double or triple seesaw case, it is technically natural that even with a seesaw mass scale as low as $1 \mathrm{TeV}$ or so, the new particles may have large couplings with the known leptons and Higgs doublets. This is generically not possible for type I or III seesaw. It is therefore an exciting possibility that some of the new particles we introduce could be produced at the LHC and detected through some distinctive decay signatures. We point out a way to experimentally distinguish the new particles involved in double seesaw from the ones in the conventional seesaw. We also consider the low energy implications of lepton-number conserving but lepton-flavor violating effective dimension six operators (in the Kähler potential).

We begin by recalling the basic structure of the NMSSM [1]. We introduce a MSSM gauge singlet chiral superfield $S$ (with even $Z_{2}$ matter parity) through the following superpotential terms:

$$
W \supset \lambda S H_{u} H_{d}+\frac{\kappa}{3} S^{3},
$$

where $\lambda$ and $\kappa$ are dimensionless constants, and $H_{u}, H_{d}$ denote the MSSM Higgs doublets. A discrete $Z_{3}$ symmetry under which $\mathrm{S}$ carries unit charge $\omega=e^{i 2 \pi / 3}$ is introduced in order to eliminate from $W$ terms that are linear and quadratic in $S$, as well as the MSSM $\mu$ term. Note that $S$ could be assigned a $Z_{3}$ charge $\omega^{2}$, but this leads to the same dimension six and seven operators for neutrino masses. In order to decide on the $Z_{3}$ charges of the MSSM Higgs doublets, we require the presence in $W$ of Yukawa couplings at the renormalizable level. There 


\begin{tabular}{|c|c|c|c|c|c|c|c|c|c|}
\hline & & $Q$ & $U^{c}$ & $D^{c}$ & $L$ & $E^{c}$ & $H_{u}$ & $H_{d}$ & $S$ \\
\hline case Ia & $Z_{3}$ & $\omega^{2}$ & 1 & 1 & $\omega^{2}$ & 1 & $\omega$ & $\omega$ & $\omega$ \\
\hline case Ib & $Z_{3}$ & 1 & $\omega$ & 1 & $\omega$ & $\omega^{2}$ & $\omega^{2}$ & 1 & $\omega$ \\
\hline case Ic & $Z_{3}$ & 1 & 1 & $\omega$ & 1 & $\omega$ & 1 & $\omega^{2}$ & $\omega$ \\
\hline \hline case IIa & $Z_{3}$ & 1 & $\omega^{2}$ & $\omega^{2}$ & 1 & $\omega^{2}$ & $\omega$ & $\omega$ & $\omega$ \\
\hline case IIb & $Z_{3}$ & 1 & $\omega$ & 1 & $\omega^{2}$ & $\omega$ & $\omega^{2}$ & 1 & $\omega$ \\
\hline case IIc & $Z_{3}$ & 1 & 1 & $\omega$ & $\omega$ & 1 & 1 & $\omega^{2}$ & $\omega$ \\
\hline \hline case IIIa & $Z_{3}$ & $\omega$ & $\omega$ & $\omega$ & $\omega$ & $\omega$ & $\omega$ & $\omega$ & $\omega$ \\
\hline case IIIb & $Z_{3}$ & 1 & $\omega$ & 1 & 1 & 1 & $\omega^{2}$ & 1 & $\omega$ \\
\hline case IIIc & $Z_{3}$ & 1 & 1 & $\omega$ & $\omega^{2}$ & $\omega^{2}$ & 1 & $\omega^{2}$ & $\omega$ \\
\hline
\end{tabular}

Table 1: $Z_{3}$ charge assignments of the NMSSM superfields which correspond to dimension five, six or seven effective operators for neutrino masses.

are several possible $Z_{3}$ charge assignments for the matter superfields that are consistent with this requirement as displayed in Table 1 . The $Z_{3}$ charge assignments in Table 1 for the quark superfields is not unique, but this will not be relevant for the discussion which follows.

We assume that the breaking of supersymmetry in the 'hidden' sector induces electroweak scale soft scalar mass terms consistent with $Z_{3}$ and $Z_{2}$ :

$$
V_{\text {soft }}=m_{H_{u}}^{2}\left|H_{u}\right|^{2}+m_{H_{d}}^{2}\left|H_{d}\right|^{2}+m_{S}^{2}|S|^{2}+\left(m_{\lambda} S H_{u} H_{d}+m_{\kappa} S^{3}+\text { h.c. }\right) .
$$

The scalar component of $S$ acquires a non-zero VEV which generates the desired MSSM $\mu$ term. The radiative electroweak breaking scenario proceeds as in the MSSM case.

According to the charge assignments in Table 1, neutrino masses arise from effective dimension five, six or seven operators:

$$
\begin{array}{ll}
\text { case Ia-Ic: } & \frac{L L H_{u} H_{u}}{M_{5}}, \\
\text { case IIa-IIc: } & \frac{L L H_{u} H_{u} S}{M_{6}^{2}}, \\
\text { case IIIa-IIIc: } & \frac{L L H_{u} H_{u} S^{2}}{M_{7}^{3}},
\end{array}
$$

where $M_{5,6,7}$ denote the appropriate seesaw mass scales.

\section{Case I}

Cases Ia-Ic correspond to the conventional dimensional five neutrino operators. According to the three distinct ways to contract the $\mathrm{SU}(2)$ indices in Eq. (3) , there are three kinds of seesaw mechanisms: type I seesaw [11] mediated by the MSSM gauge singlet fermions, type 
II seesaw [12] mediated by $\mathrm{SU}(2)$ triplet scalars with unit hypercharge, and type III seesaw [13] mediated by $\mathrm{SU}(2)$ triplet fermions with zero hypercharge. In this paper our focus will be mainly on dimension six and seven operators in Eq. (44) and Eq. (5).

\section{Case II}

Following electroweak symmetry breaking, the dimension six operator (Eq. (44)) induces light neutrino Majorana mass given by

$$
m_{\nu} \sim\left(\frac{v_{u}^{2}}{M}\right) \times\left(\frac{\langle S\rangle}{M}\right),
$$

where we set $\left\langle H_{u}\right\rangle \equiv v_{u}$. Compared to the conventional seesaw formula, we have an additional suppression factor $\langle S\rangle / M$, so for this case the upper bound on the seesaw mass scale is of order $10^{8} \mathrm{GeV}$, assuming all Yukawa couplings involved in the seesaw mechanism are of order unity. In practice, the seesaw scale can be much lower.

It is interesting and instructive to propose an explicit model which can generate these effective dimension six operators. We will show that the masses of some of the new particles we introduce can be within reach of the LHC.

Note that case IIa-IIc give rise to the same dimension six operator given by Eq. (4). The heavy fields which generate dimension six operators for case IIa-IIc will differ only in the choice of $Z_{3}$ charges. Thus, we will consider only case IIa, which is easily generalized for IIb and IIc.

As our first example on how to generate dimension six operators (Eq. (44)), we introduce the following new particles in the NMSSM (Figure 1),

\begin{tabular}{c|cc|cc} 
& $\mathrm{SU}(2)$ & $\mathrm{U}(1)$ & $Z_{3}$ & $Z_{2}$ \\
\hline$N_{j}^{c}$ & $\mathbf{1}$ & 0 & $\omega^{2}$ & - \\
$N_{j}$ & $\mathbf{1}$ & 0 & $\omega$ & -
\end{tabular}

where $i, j$ denote the generation indices. To reproduce the neutrino oscillation data, we need to introduce at least two generations of $N^{c}$ and $N$.

The renormalizable superpotential terms involving only the new chiral superfields is given by

$$
W \supset Y_{i j} N_{i}^{c}\left(H_{u} L_{j}\right)+\frac{\left(\lambda_{N}\right)_{i j}}{2} S N_{i} N_{j}+m_{i j} N_{i}^{c} N_{j}
$$

For $m_{i j}$ larger than the electroweak scale, we integrate out the heavy fields $N_{i}^{c}$ and $N_{i}$ under the SUSY vacuum conditions,

$$
\begin{aligned}
& \frac{\partial W}{\partial N}=0 \rightarrow N^{c}=-m^{-1} \lambda_{N} S N \\
& \frac{\partial W}{\partial N^{c}}=0 \rightarrow N=-m^{-1} Y\left(H_{u} L\right),
\end{aligned}
$$


where the equations are to be understood in matrix form. Thus we arrive at the dimension six operator,

$$
W_{\text {eff }}=\frac{1}{2}\left(H_{u} L\right)^{T} Y^{T}\left(m^{-1}\right)^{T}\left(\lambda_{N} S\right) m^{-1} Y\left(H_{u} L\right)
$$

For simplicity, we take $m_{i j}=M_{6} \delta_{i j}$ and $\left(\lambda_{N}\right)_{i j}=\lambda_{N} \delta_{i j}$. Following the electroweak symmetry breaking, the neutrino Majorana mass matrix is generated:

$$
m_{\nu}=\frac{\left(Y^{T} Y\right) v_{u}^{2}}{M_{6}} \times \frac{\lambda_{N}\langle S\rangle}{M_{6}} .
$$

This formula implies that even if $Y=\mathcal{O}(1)$ and $M \sim 1 \mathrm{TeV}$, the correct mass scale for the light neutrinos can be reproduced by suitably adjusting $\lambda_{N}$.

Note that the heavy fields integrated out also have an impact on the Kähler potential. Substituting Eq. (9) into the canonical Kähler potential for the heavy fields, $\int d^{4} \theta\left(N^{\dagger} N+\right.$ $N^{c \dagger} N^{c}$ ), we obtain (see Figure 2)

$$
\mathcal{K}_{\mathrm{eff}}=\left(H_{u} L\right)^{\dagger} Y^{\dagger}\left(m^{-1}\right)^{\dagger} m^{-1} Y\left(H_{u} L\right)+\cdots
$$

where the ellipsis denote higher order terms. Following the electroweak symmetry breaking, this dimension six operator induces flavor-dependent corrections to the kinetic term of the left-handed neutrinos [14]. Taking again for simplicity $m_{i j}=M_{6} \delta_{i j}$ and $\left(\lambda_{N}\right)_{i j}=\lambda_{N} \delta_{i j}$, the modified kinetic term of the left-handed neutrinos is found to be

$$
\mathcal{L}_{\text {kin }}=i \overline{\nu_{L i}}\left(\delta_{i j}+\epsilon_{i j}^{N}\right) \gamma^{\mu} \partial_{\mu} \nu_{L j}
$$

where

$$
\epsilon_{i j}^{N}=\frac{v_{u}^{2}}{M_{6}^{2}}\left(Y^{\dagger} Y\right)_{i j} .
$$

In the presence of the flavor-dependent kinetic term, the relation between the mass $(\tilde{\nu})$ and flavor $(\nu)$ eigenstates of the light Majorana neutrinos is given by $\nu_{i}=\left(\mathcal{N} U_{\mathrm{MNS}}\right)_{i j} \tilde{\nu}_{j}$, with $U_{M N S}$ the usual neutrino mixing matrix and

$$
\mathcal{N}_{i j} \simeq \delta_{i j}-\frac{1}{2} \epsilon_{i j}^{N}
$$

in the approximation $\epsilon_{i j}^{N} \ll 1$. Note that the matrix $\mathcal{N}$ is not unitary. This causes interesting modifications in both the charged and neutral currents (involving light neutrinos) in the SM:

$$
J_{\mu}^{C C}=\overline{\tilde{e}_{L i}} \gamma_{\mu} \mathcal{N}_{i j} \tilde{\nu}_{L j}, \quad J_{\mu}^{N C}=\frac{1}{2} \overline{\tilde{\nu}_{L i}} \gamma_{\mu}\left(\mathcal{N}^{\dagger} \mathcal{N}\right)_{i j} \tilde{\nu}_{L j}
$$


expressed in terms of the mass eigenstates. It turns out [15, 16] that the elements of $\left|\mathcal{N N}^{\dagger}\right|$ are somewhat severely constrained by the current experimental data on neutrino oscillations, $W$ and $Z$ boson decays, and flavor-violating decays of leptons: $Y_{i j} \lesssim 0.1$ for $M_{6} \sim 1 \mathrm{TeV}$. On the other hand, new signals of CP-violation related to this non-unitary leptonic mixing may be observed in future neutrino oscillation experiments [17].

An alternative way to generate the effective dimension six operator (Eq. (44)) is to replace the singlet superfields in Eq. (7) with two $\mathrm{SU}(2)$ triplets with zero-hypercharge:

\begin{tabular}{c|cc|cc} 
& $\mathrm{SU}(2)$ & $\mathrm{U}(1)$ & $Z_{3}$ & $Z_{2}$ \\
\hline$\Delta_{j}^{c}$ & $\mathbf{3}$ & 0 & $\omega^{2}$ & - \\
$\Delta_{j}$ & $\mathbf{3}$ & 0 & $\omega$ & -
\end{tabular}

The superpotential in this case is given by

$$
W \supset Y_{i j}\left(H_{u} \Delta_{i}^{c} L_{j}\right)+\frac{\left(\lambda_{\Delta}\right)_{i j}}{2} S \operatorname{tr}\left[\Delta_{i} \Delta_{j}\right]+m_{i j} \operatorname{tr}\left[\Delta_{i}^{c} \Delta_{j}\right]
$$

Integrating out the heavy triplets give rise to dimension six operators in the superpotential and the Kähler potential. Substituting the various VEVs, we obtain the light neutrino Majorana masses and flavor-violating kinetic terms. One difference from the previous (singlet) case is that dimension six operators are also induced for the charged leptons by integrating out the heavy charged fields in the $\mathrm{SU}(2)$ triplets. Again, in the mass basis, a non-unitary mixing matrix is induced, whose elements are constrained by the current experimental data [16]: $Y_{i j} \lesssim 0.01-0.1$ for $m_{i j} \sim 1 \mathrm{TeV}$.

Yet another way for generating the dimension six operator is to introduce four additional $\mathrm{SU}(2)$ triplets with unit hypercharge (see Figure 3):

\begin{tabular}{c|cc|cc} 
& $\mathrm{SU}(2)$ & $\mathrm{U}(1)$ & $Z_{3}$ & $Z_{2}$ \\
\hline$\Delta^{c}$ & $\mathbf{3}$ & +1 & 1 & + \\
$\bar{\Delta}^{c}$ & $\mathbf{3}$ & -1 & 1 & + \\
$\Delta$ & $\mathbf{3}$ & -1 & $\omega$ & + \\
$\bar{\Delta}$ & $\mathbf{3}$ & +1 & $\omega^{2}$ & +
\end{tabular}

The additional contributions to the NMSSM superpotential in this case contain the following terms

$$
W \supset Y_{i j}\left(L_{i} \Delta^{c} L_{j}\right)+Y_{H}\left(H_{u} \Delta H_{u}\right)+\lambda_{N} S \operatorname{tr}\left[\bar{\Delta}^{c} \bar{\Delta}\right]+m_{c} \operatorname{tr}\left[\bar{\Delta}^{c} \Delta^{c}\right]+m \operatorname{tr}[\bar{\Delta} \Delta] .
$$

In this case, the effective Kähler potential after integrating out the heavy triplets is found to be of the form,

$$
\mathcal{K}_{\text {eff }} \sim Y_{i j}^{\dagger} Y_{k l} \frac{L_{i}^{\dagger} L_{j}^{\dagger} L_{k} L_{l}}{m_{c}^{2}}
$$


such that lepton-flavor violating four-Fermi interaction is induced. The Yukawa coupling $Y_{i j}$ is constrained by the data from flavor-violating decays of leptons [16]: $Y_{i j} \lesssim 0.01-0.1$ for $m_{c} \sim 1$ $\mathrm{TeV}$.

As an example of a more elaborate model, let us introduce the following additions to the NMSSM:

\begin{tabular}{c|cc|cc} 
& $\mathrm{SU}(2)$ & $\mathrm{U}(1)$ & $Z_{3}$ & $Z_{2}$ \\
\hline$H_{u j}^{\prime}$ & $\mathbf{2}$ & $+1 / 2$ & $\omega^{2}$ & - \\
$H_{d j}^{\prime}$ & $\mathbf{2}$ & $-1 / 2$ & $\omega$ & - \\
$\Delta_{j}^{c}$ & $\mathbf{3}$ & 0 & $\omega$ & - \\
$\bar{\Delta}_{j}^{c}$ & $\mathbf{3}$ & 0 & $\omega^{2}$ & -
\end{tabular}

The relevant part of the superpotential is given by

$$
W \supset Y_{S}^{i j} S\left(H_{u i}^{\prime} L_{j}\right)+\left(\lambda_{\Delta}\right)_{i j}\left(H_{u} \Delta_{i}^{c} H_{d i}^{\prime}\right)+Y_{\Delta}^{i j}\left(H_{u} \bar{\Delta}_{i}^{c} L_{j}\right)+m_{H}^{i j}\left(H_{u i}^{\prime} H_{d j}^{\prime}\right)+m_{\Delta}^{i j} \operatorname{tr}\left[\bar{\Delta}_{i}^{c} \Delta_{j}^{c}\right] .
$$

The dimension six operators arise as shown in Figure 4. The corresponding part of the Kähler potential takes the form (symbolically),

$$
\mathcal{K}_{\mathrm{eff}} \sim Y_{S}^{\dagger} Y_{S} \frac{L^{\dagger} L S^{\dagger} S}{m_{H}^{2}}+Y_{\Delta}^{\dagger} Y_{\Delta} \frac{L^{\dagger} L H_{u}^{\dagger} H_{u}}{m_{\Delta}^{2}},
$$

and the flavor-dependent kinetic terms are generated via the VEVs of $S$ and $H_{u}$. Comparing to the constraints on $Y \mathrm{~s}$ in the previous cases, we read the current experimental bounds as $Y_{S}^{i j}, Y_{\Delta}^{i j} \lesssim 0.01-0.1$ for $m_{H}, m_{\Delta} \sim 1 \mathrm{TeV}$.

\section{Case III}

Cases IIIa-IIIc correspond to dimension seven operators for neutrino masses and mixings (see Eq. (5) ). We note that this operator can be generated by integrating out the same heavy fields which we introduced for generating the dimensional six operator. The main difference is in the $Z_{3}$ charge assignments. It is obvious that there are more possibilities to generate dimension seven operators for neutrino masses compared to the dimensional six case. We will provide one example of how to generate such an operator.

For Case IIIa, we introduce the following new particles in the NMSSM spectrum (see Figure 5),

\begin{tabular}{c|cc|cc} 
& $\mathrm{SU}(2)$ & $\mathrm{U}(1)$ & $Z_{3}$ & $Z_{2}$ \\
\hline$N_{j}^{c}$ & $\mathbf{1}$ & 0 & $\omega$ & - \\
$N_{j}$ & $\mathbf{1}$ & 0 & $\omega^{2}$ & - \\
$N_{j}^{0}$ & $\mathbf{1}$ & 0 & 1 & -
\end{tabular}


where $i, j$ denote the generation indices. To reproduce the neutrino oscillation data, we need to introduce at least two generations of $N^{c}, N^{0}$ and $N$.

The relevant part of the renormalizable superpotential involving only the new chiral superfields is given by

$$
W \supset Y_{i j} N_{i}^{c}\left(H_{u} L_{j}\right)+\left(\lambda_{N}\right)_{i j} S N_{i} N_{j}^{0}+m_{i j} N_{i}^{c} N_{j}+\frac{1}{2} m_{i j}^{\prime} N_{i}^{0} N_{j}^{0} .
$$

For $m_{i j}$ and $m_{i j}^{\prime}$ larger than the electroweak scale, we integrate out the heavy $N_{i}^{c}, N_{i}^{0}$ and $N_{i}$ under the SUSY vacuum conditions. After eliminating the heavy fields, we arrive at the dimension seven operator of the form:

$$
W_{\mathrm{eff}}=-\frac{1}{2}\left(H_{u} L\right)^{T} Y^{T}\left(m^{-1}\right)^{T}\left(\lambda_{N} S\right)^{T}\left(m^{\prime}\right)^{-1}\left(\lambda_{N} S\right) m^{-1} Y\left(H_{u} L\right)
$$

For simplicity, we take $m_{i j}=m_{i j}^{\prime}=M_{7} \delta_{i j}$ and $\left(\lambda_{N}\right)_{i j}=\lambda_{N} \delta_{i j}$. Following the electroweak symmetry breaking, the neutrino Majorana mass matrix is generated:

$$
m_{\nu}=\frac{\left(Y^{T} Y\right) v_{u}^{2}}{M_{7}} \times \frac{\lambda_{N}^{T} \lambda_{N}\left\langle S^{2}\right\rangle}{M_{7}^{2}} .
$$

We can see from this formula that the upper bound for seesaw scale is $M_{7} \sim 10^{6} \mathrm{GeV}$, assuming all Yukawa coupling in Eq. (27) are $\mathcal{O}(1)$. It is clear from Eq. (28) that by suitably adjusting the parameter $\lambda_{N}$, the seesaw scale $M_{7}$ can easily be lowered to the TeV range. This opens up the exciting possibility that some of the new particles in Eq. (26) can have $\mathcal{O}(1)$ Yukawa couplings with the known matter fields.

If some of the new particles generating the seesaw mechanism have masses around $1 \mathrm{TeV}$, they could be produced in hadron colliders [18]. In type I seesaw, the heavy Majorana neutrino productions at hadron colliders via $W$-boson exchange and $W W$-fusion process [19, 20, 21] have been investigated. Signatures for the Majorana neutrinos could be observed through their lepton-number violating decays leading to like-sign dilepton production [20, 21]. However, the production cross section is normally small, because the heavy neutrinos dominantly consist of the singlet Majorana neutrinos and the couplings between the heavy neutrinos and the $W$-boson are suppressed by a factor $Y v_{u} / M_{5}$, the mixing between left-handed neutrinos and right-handed heavy neutrinos induced by the seesaw mechanism. Agreement with the neutrino oscillation data requires that, $Y$ should at most be around $10^{-5}$, and so the mixing is very small for $M_{5} \sim 1$ $\mathrm{TeV}$. Some fine-tuning for the Dirac Yukawa matrix is necessary to keep the mixing angle as large as possible while reproducing the neutrino oscillation data [21].

The situation for type II or III seesaw is different, since the new particles involved in the seesaw mechanism have gauge couplings with the photon, $W$ and $Z$-boson. Thus, these new particles could be produced at the LHC through processes mediated by these gauge boson. 
The signature of such particles in type II seesaw [22] and in type III seesaw [23] have been investigated in detail. For example, the doubly-charged scalar in type II seesaw, once produced, may provide a clean signature through its decay into a pair of same sign charged leptons [22]. The pair production of the singly-charged fermions in type III seesaw and their (lepton-number and/or lepton-flavor violating) decays into leptons and $W, Z$-boson or Higgs boson could be discovered at the LHC. Note that if the seesaw scale is around $1 \mathrm{TeV}$, the Dirac Yukawa couplings are very small, say, $Y=\mathcal{O}\left(10^{-5}\right)$ to provide a light neutrino mass $m_{\nu}=\mathcal{O}(1 \mathrm{eV})$. This fact implies that the new particle production is accompanied by an extra signature [23]: The lifetime of the produced particles is long enough for the their decay vertices to be detectably displaced from the primary production vertex.

The new particles included in the conventional seesaw models also appear in models with the double (or triple) seesaw mechanism. The collider phenomenology for these particles is analogous to the one in the conventional seesaw models. However, there is a crucial difference arising from the structure of the double (or triple) seesaw mechanism. As we have already noted, in the double (or triple) seesaw mechanism we can reproduce the light neutrino Majorana masses while keeping the Dirac Yukawa couplings as large as possible, so long as the couplings among the new particles are suitably small. Therefore, the production cross section of the heavy neutrinos in type I seesaw can be sizable. If the Dirac Yukawa couplings are much larger than the values expected in the conventional seesaw models with a $\mathrm{TeV}$ seesaw scale, the lifetimes of the new particles can be relatively short. For a low seesaw scale this could be a distinguishing feature between the conventional seesaw and the double (or triple) seesaw models. In addition, we have seen that in models with double (or triple) seesaw, new SU(2) doublets, which do not appear in the conventional seesaw models, can be present. The phenomenology of these new particles would be worth investigating. For example, the charged scalar in the doublet superfield $\left(H_{u}^{\prime}\right)$, once produced, can decay into charged-leptons and the fermionic component of the singlet superfield $S$.

Recently, it has been pointed out [8] that if the right-handed neutrinos have couplings with the singlet $\mathrm{S}$ in the NMSSM, the lightest right-handed sneutrino can be a viable cold dark matter candidate through its coupling with the Higgs bosons. Our double (or triple) seesaw mechanism with right-handed neutrinos shares the same structure for the right-handed sneutrinos, so that this scenario can also work in our models.

Finally, let us recall that the presence of low scale seesaw can alter the predictions for the SM Higgs boson mass [24]. In the NMSSM case, the coupling $Y_{H}\left(H_{u} \Delta H_{u}\right)$ in Eq. (20) will generate a tree level contribution to the lightest CP-even Higgs boson mass [25]:

$$
m_{h}^{2}=\left(m_{h}^{2}\right)_{N M S S M}+4 Y_{H}^{2} v_{u}^{2} \sin ^{2} \beta
$$


where $\tan \beta=v_{u} / v_{d}$ and $\left(m_{h}^{2}\right)_{N M S S M}$ denotes the standard NMSSM contribution. There is a constraint on the triplet Higgs VEV arising from a global fit of electroweak data [26]:

$$
v_{\Delta} \lesssim 2 \mathrm{GeV}
$$

If one assumes that the triplet Higgs mass is a few $\mathrm{TeV}$, the trilinear coupling $Y_{H}$ can be order unity. In this case the tree level contribution can help to make the Higgs mass as heavy as $200 \mathrm{GeV}$ (for $\tan \beta>10$ ). For a more precise estimate, loop correction arising from the Higgs triplet should be included.

In summary, we have proposed extensions of the NMSSM particle spectrum such that the observed neutrino masses are described in terms of effective dimension six or seven (instead of dimension five) operators. In addition to the models corresponding to type I, II and III seesaw, we introduce more exotic possibilities with $\mathrm{SU}(2)$ doublet superfields which do not appear in the conventional seesaw models. The new heavy states responsible for the seesaw mechanism can have sizable couplings to the known leptons and may be detected at the LHC. The low energy implications of lepton flavor violating dimension six operators in the Kähler potential are briefly discussed.

\section{Acknowledgments}

N.O. would like to thank the Particle Theory Group of the University of Delaware for hospitality during his visit. He would also like to thank the Maryland Center for Fundamental Physics, and especially Rabindra N. Mohapatra for their hospitality and financial support during his stay. This work is supported in part by the DOE Grant \# DE-FG02-91ER40626 (I.G. and Q.S.), GNSF grant 07_462_4-270 (I.G.), the National Science Foundation Grant No. PHY-0652363 (N.O.), and the Grant-in-Aid for Scientific Research from the Ministry of Education, Science and Culture of Japan, \#18740170 (N.O.).

\section{References}

[1] P. Fayet, Nucl. Phys. B 90 (1975) 104; H. P. Nilles, M. Srednicki and D. Wyler, Phys. Lett. B 120 (1983) 346; J. P. Derendinger and C. A. Savoy, Nucl. Phys. B 237 (1984) 307; J. Ellis, J. F. Gunion, H. E. Haber, L. Roszkowski and F. Zwirner, Phys. Rev. D 39 (1989) 844; L. Durand and J. L. Lopez, Phys. Lett. B 217 (1989) 463; M. Drees, Int. J. Mod. Phys. A 4 (1989) 3635.

[2] U. Ellwanger, Phys. Lett. B 303 (1993) 271; U. Ellwanger, M. Rausch de Traubenberg and C. A. Savoy, Phys. Lett. B 315 (1993) 331; T. Elliott, S. F. King and P. L. White, 
Phys. Lett. B351 (1995) 213; S. F. King and P. L. White, Phys. Rev. D52 (1995) 4183 ; G. K. Yeghian, arXiv:hep-ph/9904488; U. Ellwanger and C. Hugonie, Mod. Phys. Lett. A 22, 1581 (2007).

[3] M. Bastero-Gil, C. Hugonie, S. F. King, D. P. Roy and S. Vempati, Phys. Lett. B 489, 359 (2000).

[4] R. Dermisek and J. F. Gunion, Phys. Rev. Lett. 95, 041801 (2005).

[5] B. A. Dobrescu and K. T. Matchev, JHEP 0009, 031 (2000).

[6] K. Cheung, J. Song and Q. S. Yan, Phys. Rev. Lett. 99, 031801 (2007).

[7] D. G. Cerdeno, C. Hugonie, D. E. Lopez-Fogliani, C. Munoz and A. M. Teixeira, JHEP 0412, 048 (2004); JCAP 0706, 008 (2007); J. F. Gunion, D. Hooper and B. McElrath, Phys. Rev. D 73, 015011 (2006).

[8] D. G. Cerdeno, C. Munoz and O. Seto, arXiv:0807.3029 [hep-ph].

[9] B. T. Cleveland et.al, Astrophys.J. 496505 (1998); Super-Kamiokande Collaboration, Phys. Lett. B539 179 (2002); Super-Kamiokande Collaboration, Phys. Rev. D71 112005 (2005); M. Maltoni, T. Schwetz, M.A. Tortola, J.W.F. Valle New J.Phys. 6122 (2004); A. Bandyopadhyay et al, Phys. Lett. B608 115 (2005); G. L. Fogli et al, Prog. Part. Nucl. Phys. 57742 (2006); For a recent review, see, for example, H. Nunokawa, S. J. Parke and J. W. F. Valle, Prog. Part. Nucl. Phys. 60, 338 (2008).

[10] R. N. Mohapatra, Phys. Rev. Lett. 56 (1986) 561; R. N. Mohapatra and J. W. F. Valle, Phys. Rev. D 34, 1642 (1986); S. Nandi and U. Sarkar, Phys. Rev. Lett. 56, 564 (1986).

[11] P. Minkowski, Phys. Lett. B 67, 421 (1977); T. Yanagida, in Proceedings of the Workshop on the Unified Theory and the Baryon Number in the Universe (O. Sawada and A. Sugamoto, eds.), KEK, Tsukuba, Japan, 1979, p. 95; M. Gell-Mann, P. Ramond, and R. Slansky, Supergravity (P. van Nieuwenhuizen et al. eds.), North Holland, Amsterdam, 1979, p. 315; S. L. Glashow, The future of elementary particle physics, in Proceedings of the 1979 Cargèse Summer Institute on Quarks and Leptons (M. Levy et al. eds.), Plenum Press, New York, 1980, p. 687; R. N. Mohapatra and G. Senjanovic, Phys. Rev. Lett. 44, 912 (1980).

[12] G. Lazarides, Q. Shafi and C. Wetterich, Nucl. Phys. B181, 287 (1981); R. N. Mohapatra and G. Senjanovic, Phys. Rev. D 23, 165 (1981); M. Magg and C. Wetterich, Phys. Lett. B 94, 61 (1980); J. Schechter and J. W. F. Valle, Phys. Rev. D 22, 2227 (1980). 
[13] R. Foot, H. Lew, X. G. He and G. C. Joshi, Z. Phys. C 44, 441 (1989).

[14] A. Broncano, M. B. Gavela and E. E. Jenkins, Phys. Lett. B 552, 177 (2003) [Erratum-ibid. B 636, 330 (2006)]; Nucl. Phys. B 672, 163 (2003).

[15] S. Antusch, C. Biggio, E. Fernandez-Martinez, M. B. Gavela and J. Lopez-Pavon, JHEP 0610, 084 (2006).

[16] A. Abada, C. Biggio, F. Bonnet, M. B. Gavela and T. Hambye, JHEP 0712, 061 (2007).

[17] E. Fernandez-Martinez, M. B. Gavela, J. Lopez-Pavon and O. Yasuda, Phys. Lett. B 649, 427 (2007).

[18] For detailed LHC studies regarding the three seesaw scenarios see F. del Aguila and J. A. Aguilar-Saavedra, arXiv:0808.2468 [hep-ph].

[19] A. Pilaftsis, Z. Phys. C 55, 275 (1992).

[20] D. A. Dicus, D. D. Karatas and P. Roy, Phys. Rev. D 44, 2033 (1991). A. Datta, M. Guchait and D. P. Roy, Phys. Rev. D 47, 961 (1993); A. Datta, M. Guchait and A. Pilaftsis, Phys. Rev. D 50, 3195 (1994); A. Ferrari et al., Phys. Rev. D 62, 013001 (2000); F. M. L. Almeida, Y. D. A. Coutinho, J. A. Martins Simoes and M. A. B. do Vale, Phys. Rev. D 62, 075004 (2000); O. Panella, M. Cannoni, C. Carimalo and Y. N. Srivastava, Phys. Rev. D 65, 035005 (2002); T. Han and B. Zhang, Phys. Rev. Lett. 97, 171804 (2006); F. del Aguila, J. A. Aguilar-Saavedra and R. Pittau, JHEP 0710, 047 (2007).

[21] J. Kersten and A. Y. Smirnov, Phys. Rev. D 76, 073005 (2007).

[22] K. Huitu, J. Maalampi, A. Pietila and M. Raidal, Nucl. Phys. B 487, 27 (1997); E. J. Chun, K. Y. Lee and S. C. Park, Phys. Lett. B 566, 142 (2003); A. G. Akeroyd and M. Aoki, Phys. Rev. D 72, 035011 (2005); T. Han, B. Mukhopadhyaya, Z. Si, and K. Wang, Phys. Rev. D76, 075013 (2007); J. Garayoa and T. Schwetz, arXiv:0712.1453 [hep-ph]; A. G. Akeroyd, M. Aoki and H. Sugiyama, arXiv:0712.4019 [hep-ph]; P. Fileviez Perez, T. Han, G. y. Huang, T. Li and K. Wang, Phys. Rev. D 78, 015018 (2008).

[23] B. Bajc and G. Senjanovic, JHEP 0708, 014 (2007); B. Bajc, M. Nemevsek and G. Senjanovic, Phys. Rev. D 76, 055011 (2007); R. Franceschini, T. Hambye and A. Strumia, Phys. Rev. D 78, 033002 (2008).

[24] I. Gogoladze, N. Okada and Q. Shafi, arXiv:0802.3257 [hep-ph]; arXiv:0805.2129 [hep-ph]. 
[25] J. R. Espinosa and M. Quiros, Phys. Rev. Lett. 81, 516 (1998).

[26] F. del Aguila, J. A. Aguilar-Saavedra, J. de Blas and M. Perez-Victoria, arXiv:0806.1023 [hep-ph]. 


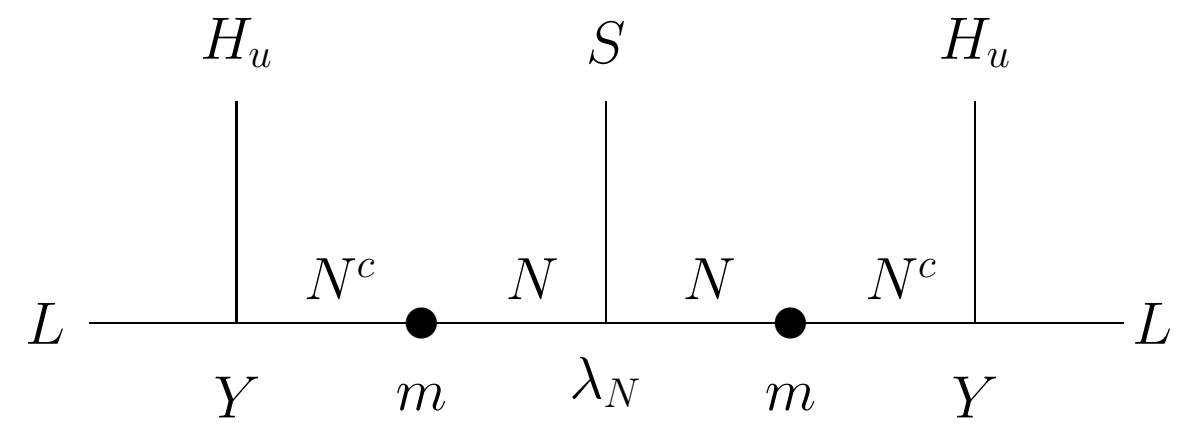

Figure 1: Supergraph leading to dimension six operator in the superpotential of Eq. (10).

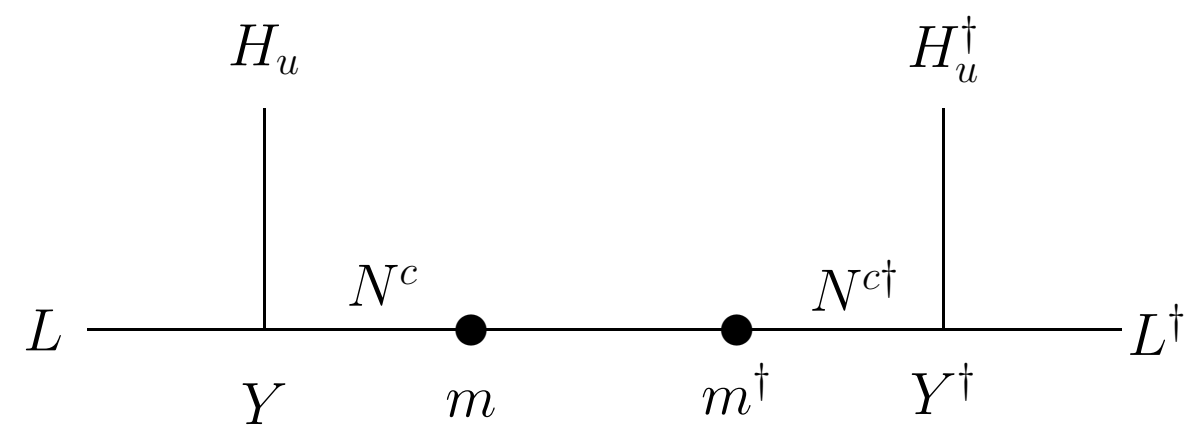

Figure 2: Supergraph leading to dimension six operator in the Kähler potential of Eq. (12). 


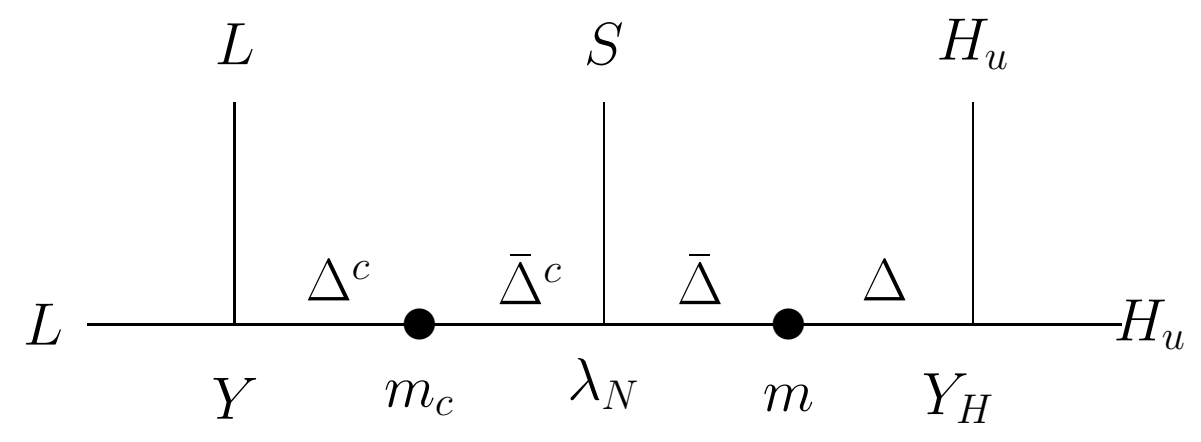

Figure 3: Supergraph leading to dimension six operator after integrating out the heavy triplets.

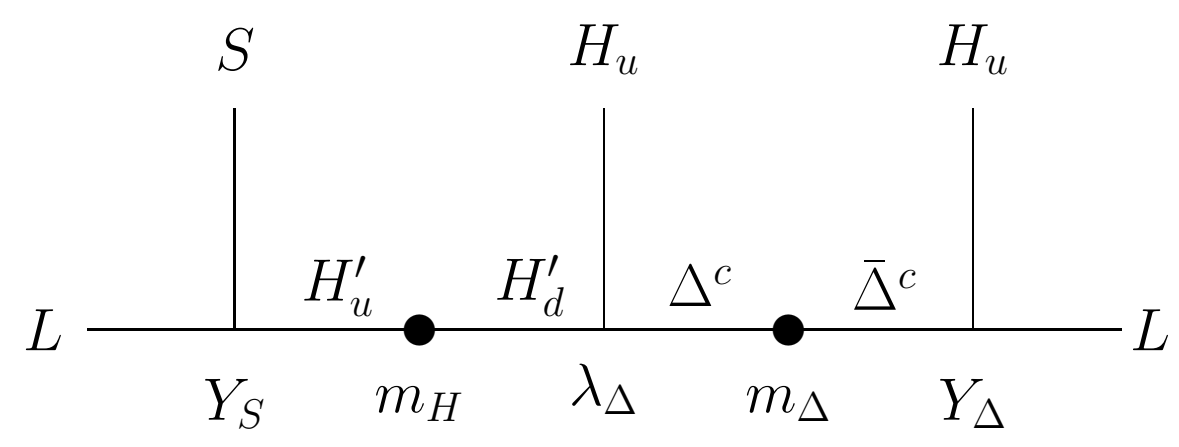

Figure 4: Supergraph with additional doublets leading to dimension six operator. 


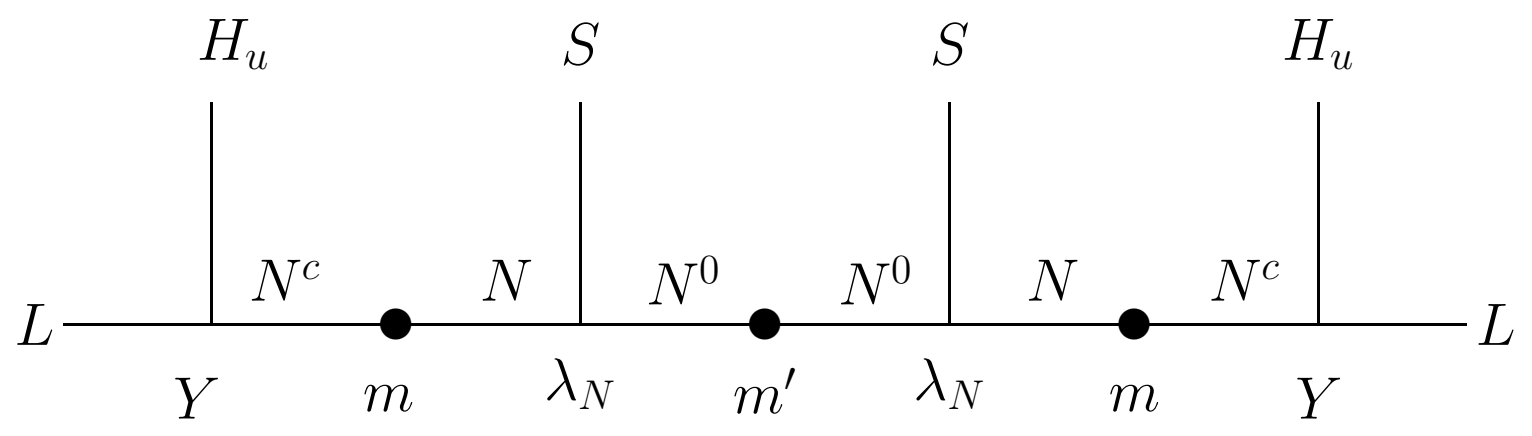

Figure 5: Supergraph leading to dimension seven operator in the superpotential of Eq. (27). 